\title{
س
}

$>\mathrm{DE}$

$\cong$ PÉDAGOGIE

\author{
Recherches en éducation
}

168 | juillet-septembre 2009

Enseignement et apprentissages, entre psychologie et didactiques

\section{CARLIER Michèle \& AYOUN Catherine. Déficiences intellectuelles et intégration sociale}

Wavre : Mardaga, 2007. - 221 p.

Daniel Mellier

\section{(2) OpenEdition}

Journals

Édition électronique

URL : http://journals.openedition.org/rfp/1820

DOI : $10.4000 / \mathrm{rfp} .1820$

ISSN : 2105-2913

Éditeur

ENS Éditions

Édition imprimée

Date de publication : 1 juillet 2009

Pagination : 138-139

ISBN : 978-2-7342-1172-3

ISSN : 0556-7807

Référence électronique

Daniel Mellier, «CARLIER Michèle \& ayoun Catherine. Déficiences intellectuelles et intégration sociale », Revue française de pédagogie [En ligne], 168 | juillet-septembre 2009, mis en ligne le 07 octobre 2010, consulté le 24 septembre 2020. URL : http://journals.openedition.org/rfp/1820 ; DOI : https://doi.org/ $10.4000 /$ rfp. 1820

Ce document a été généré automatiquement le 24 septembre 2020

(c) tous droits réservés 


\section{CARLIER Michèle \& AYOUN Catherine. Déficiences intellectuelles et intégration sociale}

Wavre : Mardaga, 2007. - 221 p.

Daniel Mellier

\section{RÉFÉRENCE}

CARLIER Michèle \& AYOUN Catherine. Déficiences intellectuelles et intégration sociale. Wavre :

Mardaga, 2007. - $221 \mathrm{p}$.

1 Le livre de Michèle Carlier et Catherine Ayoun vient à point nommé quand la question de la déficience intellectuelle est revisitée à la lumière des découvertes génétiques. Il apporte en effet aux parents, aux étudiants comme aux praticiens des informations claires et utiles pour ne pas se perdre dans les concepts des sciences de l'hérédité. Il propose par ailleurs, après en avoir discuté l'intérêt et les limites, les portraits psychologiques ou "phénotypes comportementaux" des personnes porteuses des anomalies les plus connues. Il donne enfin des informations pratiques pour se repérer dans le dédale des pratiques institutionnelles et administratives qui concourent à l'intégration sociale scolaire des personnes en situation de handicap. Le livre est ouvert par un historique, succinct mais éclairant, de l'évolution des pratiques sociales envers les personnes déficientes intellectuelles. On y comprend comment ces pratiques, aujourd'hui jugées barbares, reflètent des modes de compréhension qui sont loin de reconnaître le caractère social et d'humanité de la personne différente. On y trouve, et cela est bienvenu, les adresses des sites Internet où peuvent être lus en direct les livres des grands pédagogues qui ont contribué à l'avancée de la pensée vers les schémas actuels.

2 Le chapitre suivant résume les notions de QI et de mesure de l'efficience intellectuelle ainsi que les modèles contemporains du handicap. Les auteurs soulignent avec à-propos 
que les nouvelles éditions du WISC 4, qui ne prévoient plus le calcul du QI, ne règlent pas pour autant, ou posent autrement, la question de la définition de la déficience.

Le chapitre III expose les notions de génétique utiles pour comprendre les différentes formes de déficiences associées aux anomalies de ce type. Il donne aussi un état des connaissances sur les effets de prise d'alcool pendant la grossesse en rassemblant les données récentes sur le «syndrome d'alcoolisation fœtale ».

4 Les chapitres suivants sont successivement consacrés à la phénylcétonurie (erreur du métabolisme) qui induit une déficience intellectuelle si un régime alimentaire sévère n'est pas respecté. Outre les données scientifiques les mieux établies qui sont ici exposées, on apprécie aussi de comprendre l'importance du prélèvement sanguin opéré dans les trois jours après la naissance des bébés. On passe ensuite aux déficiences liées aux anomalies du chromosome X, dont le syndrome de l'X fragile, pour lesquelles les auteurs résument les connaissances quant au phénotype comportemental, tout en reconnaissant la part de différences individuelles. Le chapitre $\mathrm{v}$ présente le syndrome de Williams-Beuren et celui de Di George en explicitant les résultats des travaux récents sur le développement émotionnel et social ainsi que les psychopathologies les plus souvent associées à ces syndromes. Le chapitre vi est consacré à la trisomie 21 en restituant les données historiques d'isolation du syndrome puis en rendant compte des originalités du développement sur les plans de la latéralité, de la cognition. La question du stéréotype social de la trisomie 21 est abordée en soulignant les lacunes nombreuses dans les connaissances en ce domaine pourtant essentiel pour envisager la participation sociale des personnes déficientes intellectuelles.

Le dernier chapitre concerne l'intégration sociale avec une mention particulière pour l'insertion dans le milieu scolaire. La présentation des enjeux de la loi de 2005 et des modalités d'accompagnement des familles répond, on peut le souhaiter, aux attentes des étudiants encore peu experts et des familles qui entrent dans ce domaine particulier des réponses aux enfants à besoins spécifiques. En résumé le livre constitue une aide efficace à la compréhension de la déficience intellectuelle à un moment où les théories de référence sont contestées, à tout le moins remises en question.

\section{AUTEURS}

DANIEL MELLIER

PSY-NCA, université de Rouen 